This item was submitted to Loughborough's Research Repository by the author.

Items in Figshare are protected by copyright, with all rights reserved, unless otherwise indicated.

\title{
CPW-fed cavity-backed slot radiator loaded with an AMC reflector
}

PLEASE CITE THE PUBLISHED VERSION

http://dx.doi.org/10.1109/TAP.2011.2173152

PUBLISHER

(c) IEEE

VERSION

AM (Accepted Manuscript)

LICENCE

CC BY-NC-ND 4.0

REPOSITORY RECORD

Joubert, J., J.C. Vardaxoglou, W.G. Whittow, and J.W. Odendaal. 2019. "Cpw-fed Cavity-backed Slot Radiator Loaded with an AMC Reflector”. figshare. https://hdl.handle.net/2134/9998. 
This item was submitted to Loughborough's Institutional Repository (https://dspace.lboro.ac.uk/) by the author and is made available under the following Creative Commons Licence conditions.

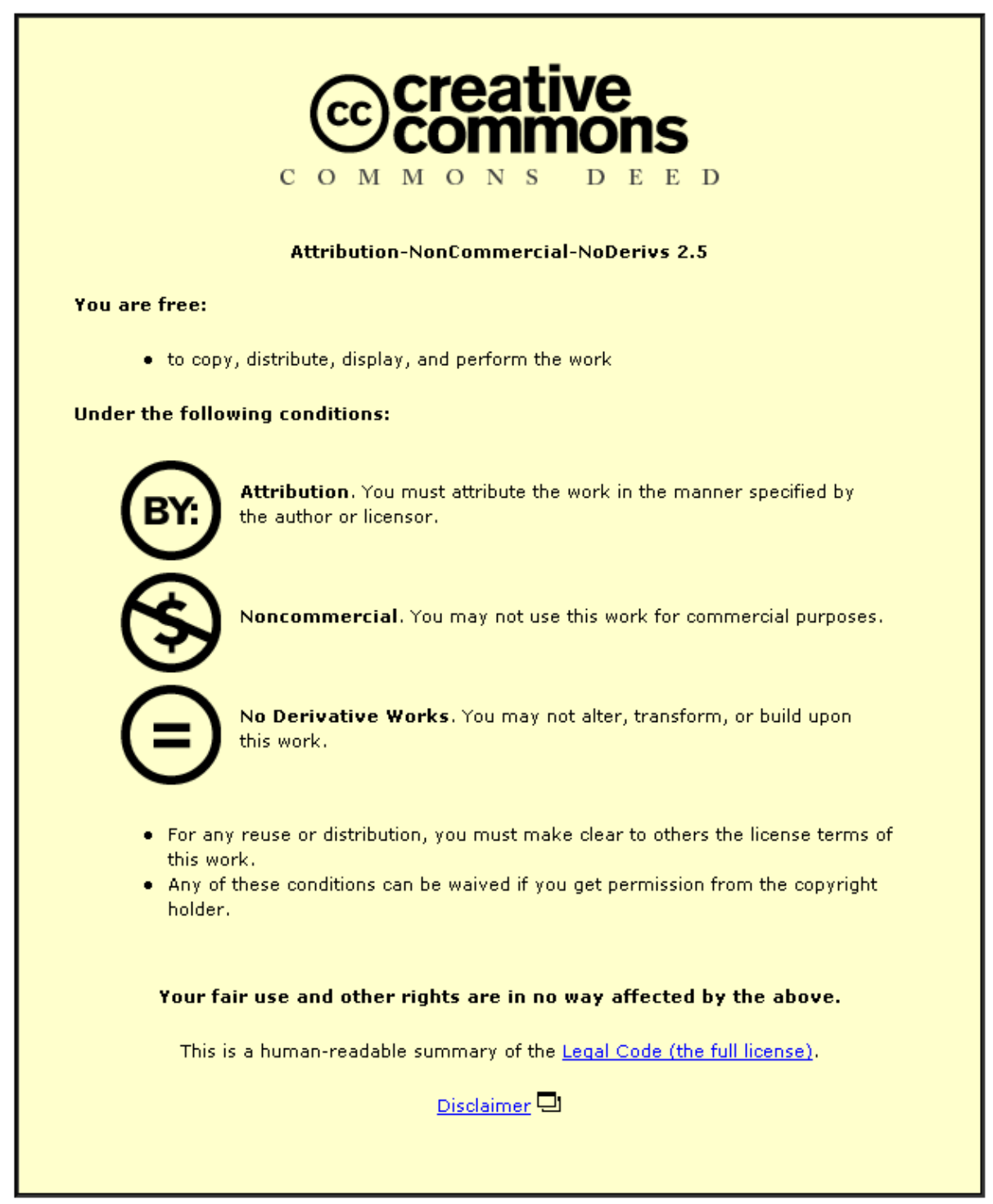

For the full text of this licence, please go to: http://creativecommons.org/licenses/by-nc-nd/2.5/ 


\title{
CPW-Fed Cavity-Backed Slot Radiator Loaded with an AMC Reflector
}

\author{
Johan Joubert, Senior Member, IEEE, J.(Yiannis) C. Vardaxoglou, Senior Member, IEEE, William G. \\ Whittow and Johann W. Odendaal, Senior Member, IEEE
}

\begin{abstract}
A low profile coplanar waveguide (CPW) fed printed slot antenna is presented with uni-directional radiation properties. The slot antenna radiates above a closely spaced artificial magnetic conducting (AMC) reflector consisting of an array of rectangular patches, a substrate and an electric ground plane. The electromagnetic band-gap (EBG) performance of the cavity structure between the upper conducting surface in which the slot is etched, and the ground plane at the bottom of the reflector, is investigated using an equivalent waveguide feed in the place of a half-wavelength section of the slot antenna. From the reflection coefficient of the equivalent waveguide feed one can determine the frequency band where minimum energy will be lost due to unwanted radiation from the cavity sides. The dimensions of the cavity were found to be very important for minimum energy loss. Experimental results for the final antenna design (with a size of $1.02 \lambda_{0} \times 0.82 \lambda_{0} \times 0.063 \lambda_{0}$ ), mounted on a $1.5 \lambda_{0} \times 1.5 \lambda_{0}$ back plate, exhibit a $5 \%$ impedance bandwidth, maximum gain in excess of $10 \mathrm{dBi}$, low cross-polarization, and a front-to-back ratio of approximately $25 \mathrm{~dB}$. This low-profile antenna with relatively high gain could be a good candidate for a 2.4 GHz WLAN application.
\end{abstract}

Index Terms-Electromagnetic band-gap materials, periodic structures, slot antennas.

\section{INTRODUCTION}

$\mathrm{S}$ OT radiators are suitable candidates for portable units and unobtrusive base stations of mobile communications systems, because of their compactness, flush-mounting and simple structure [1]. Slot radiators are also attractive when an antenna has to be integrated into a metallic surface eg. as single elements or arrays conformal to an airborne structure for electronic warfare applications [2]. When a slot is printed on one side of a substrate, the element will radiate bidirectionally, and an electric conducting surface has to be added at a distance of a quarter-wavelength below the slot as a

Manuscript received May 3, 2011.

J. Joubert and J. W. Odendaal are with the Centre for Electromagnetism, University of Pretoria, Department of Electrical, Electronic and Computer Engineering, Pretoria, 0002, South Africa (e-mail: jjoubert@up.ac.za and wimpie.odendaal@up.ac.za).

J. C. Vardaxoglou and W. G. Whittow are with the Centre for Mobile Communications Research, Department of Electronic and Electrical Engineering, Loughborough University, Loughborough LE11 3TU, U.K. (email: j.c.vardaxoglou@lboro.ac.uk andw.g.whittow@lboro.ac.uk). reflector to achieve optimum uni-directional radiation. If the reflector distance is reduced to achieve a lower profile, which is highly desirable for conformal antennas in most cases, parallel plate modes will be excited that can cause significant energy leakage. A fair amount of work was published on socalled conductor-backed CPW-fed slot antennas, where single or multiple dielectric layers were inserted between the upper conducting surface containing the slot and the reflector [3-6]. Energy leakage between the parallel plates because of unwanted modes remains a problem for conductor-backed slot radiators and special techniques have to be used to overcome this, eg. the use of closed cavities behind the slot [1], the placement of shorting pins around the slot [2], or the use of twin slot configurations for phase cancellation $[5,6]$.

This paper presents results of an investigation of a CPWfed slot radiator above a metamaterial-based AMC surface as a reflector. The intention was to investigate a single radiating element with a low profile and compact lateral dimensions, in order to improve on results for a similar structure investigated in [7]. Fig. 1 shows the geometry of the proposed structure. It can also be described as a cavity-backed (with open sides) slot radiator loaded with an AMC reflector. An alternative description would be that the radiation is due to the "structural resonance mode" [8] of the entire slot-fed cavity structure loaded with a high-impedance surface. The most important design considerations are:

1) the reflection phase of the AMC reflector - at the centre frequency it should ideally be $0^{\circ}$, and minimum variation as function of frequency will ensure optimum bandwidth of the antenna - the basic principle being that the reflected wave from the reflector should add sufficiently in phase with the resonant slot field in order to achieve a radiation resistance that can be matched to the CPW feed line,

2) the EBG performance of the physical structure between the upper conducting surface in which the slot is etched, and the ground plane at the bottom of the AMC - the propagation of any modes between the two conducting surfaces (which effectively form the parallel plate cavity structure), and subsequent radiation from the edges of the structure, should be minimized over the bandwidth of operation.

Results from a number of studies related to the topic of low profile slot antennas with a metamaterial-based reflector to 
Final Author version. Paper published in IEEE Transactions on Antennas \& Propagation. Feb. 2012, Vol. 60. Issue 2, pp. 735-742

achieve uni-directional radiation have been published in the open literature by other researchers, all reporting some level of success. One has to distinguish between compact cavitybacked slot antennas and single slot radiators embedded in a laterally large parallel plate environment. The effectiveness of the EBG structure used between the plates should be a direct function of the size of the surface area over which the structure is employed around the radiating slot - the larger the area the more effective the EBG surface. As far back as 1999 Shumpert et al [9] published results of a conductor-backed folded slot (fed with a coaxial transmission line) with an EBG structure consisting of an array of square dielectric cylinders between the plates. They achieved uni-directional radiation for a single folded slot with a front-to-back ratio of $15 \mathrm{~dB}$ and a frequency bandwidth of $7.5 \%$. The dimensions of the structure were $4 \lambda_{0} \times 4 \lambda_{0} \times 0.135 \lambda_{0}$ - so the thickness of the antenna was still more than $\lambda_{0} / 8$, and several wavelengths of the EBG structure were used to achieve significant suppression of the dominant TEM mode between the parallel plates.

Elek et al [10] published results for a uni-directional ringslot antenna. A primary focus of this work was the design of a suitable EBG surface to minimize radiation leakage between the parallel plates of the structure. They opted to use the popular "mushroom" periodic surface (small rectangular patches with vias connecting the centre of all the patches to a common ground plane) proposed by Sievenpiper et al in [11]. This work [10] contains interesting details of an experimental set-up to determine the EBG stopband. They effectively measure/simulate the transmission coefficient through a section of parallel plate waveguide with and without the EBG surface between the parallel plates, and show conclusively that the inclusion of the EBG surface dramatically suppresses the propagation of the unwanted TEM-mode between the plates. The EBG surface that is closely spaced (much closer than a quarter-wavelength) to the ground plane in which the ring-slot is cut also acts as a quasi artificial magnetic reflector (independent analysis of the structure has indicated a reflection phase close to $90^{\circ}$ at the centre frequency), which allows for a low profile structure with uni-directional radiation. The final ring-slot antenna design had overall dimensions of $2.6 \lambda_{0} \times 2.6 \lambda_{0} \times 0.08 \lambda_{0}$, but they used an EBG surface of $1.3 \lambda_{0} \times 1.3 \lambda_{0}$ centrally located behind the slot. The overall structure was still quite large laterally (with the slot asymmetrically spaced relative to the antenna ground plane without any motivation), and achieved a good front-to-back ratio of $21 \mathrm{~dB}$, and a gain improvement between 2.5 and 2.9 $\mathrm{dB}$ (compared to a ring-slot antenna without a reflector) over a $5 \%$ impedance bandwidth.

Niyomjan and Huang [12] theoretically (no experimental results were presented) investigated a suspended microstrip fed slot antenna on a high impedance surface (HIS), which is just an alternative description of an artificial magnetic conductor. Similar to [10], they also employed the Sievenpiper [11] "mushroom" periodic surface. Their final design had lateral dimensions of approximately $2 \lambda_{0} \times 2 \lambda_{0}$, and they used a relatively large gap of $4 \mathrm{~mm}\left(\approx 0.13 \lambda_{0}\right.$ at $\left.10 \mathrm{GHz}\right)$ between the slot substrate and the AMC surface, which together with the two substrate thicknesses results in a final antenna that is not significantly lower in profile than what can be achieved with a quarter-wavelength spaced electric conducting surface.

An example of a compact cavity-backed (with open sides) slot antenna (offset-fed with a microstrip line) with an etched or so-called "uni-planar compact photonic band-gap" (UCPBG) reflector was introduced by Park et al [7]. The UCPBG reflector, consisting of a lattice of square pads, capacitive gaps and narrow lines connecting each cell was also designed to act as a magnetic reflector at the resonant frequency. The dimensions of the structure were very compact and extremely thin $\left(0.62 \lambda_{0} \times 0.52 \lambda_{0} \times 0.03 \lambda_{0}\right)$. The measured performance of this antenna was however not very good, with a small experimental frequency bandwidth of $1.33 \%$, a frontto-back ratio in the vicinity of $10 \mathrm{~dB}$, poor cross polarization, and only a $1 \mathrm{~dB}$ gain improvement with reference to a slot antenna without a reflector.

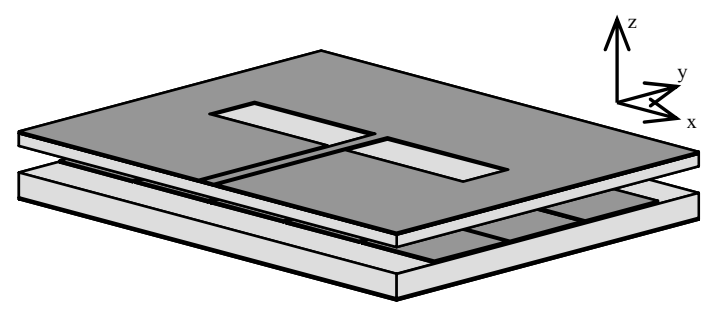

(a)

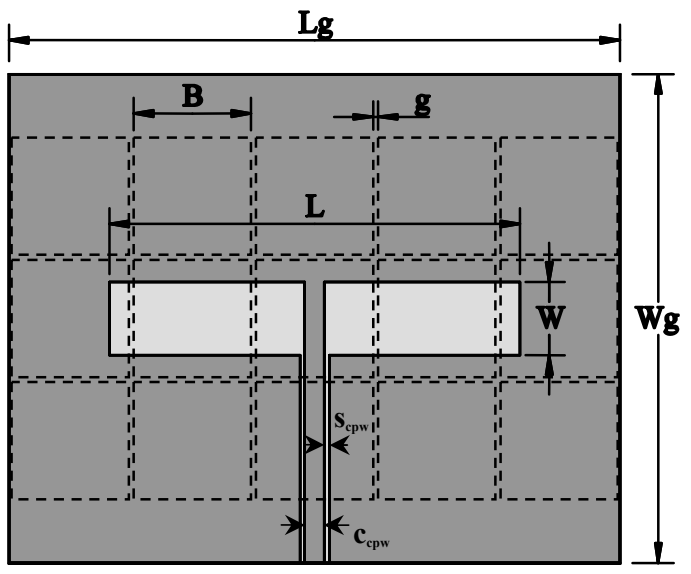

(b)

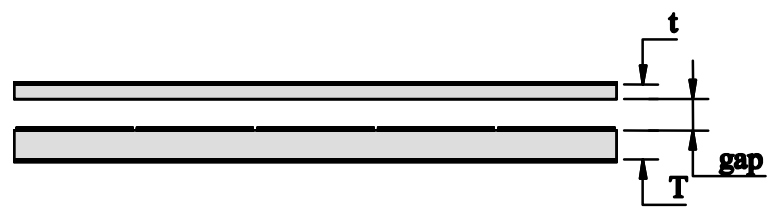

(c)

Fig. 1. Printed CPW-fed slot antenna above an AMC reflector; (a) 3D view, (b) top view, and (c) front view. 
Final Author version. Paper published in IEEE Transactions on Antennas \& Propagation. Feb. 2012, Vol. 60. Issue 2, pp. 735-742

It was decided to design the newly proposed low profile slot antenna with an AMC reflector for a practical frequency band $(2.4-2.484 \mathrm{GHz})$ - that for WLAN base stations. Section II gives details on the design of the AMC reflector consisting of an array of rectangular patches, a substrate and an electric ground plane. The cavity-backed CPW-fed slot antenna design and the EBG performance of the cavity structure (between the upper conducting surface in which the slot is etched and the ground plane at the bottom of the reflector) are discussed in section III. The EBG performance is investigated using an equivalent waveguide feed for one half-wavelength section of the slot antenna. From the waveguide reflection coefficient a designer can determine the frequency band where minimum energy will be lost due to unwanted modes radiating from the cavity sides. The dimensions of the cavity were found to be very important for minimum energy loss. Simulated and experimental results for the whole structure are presented in section IV.

\section{AMC REFLECTOR DESIGN}

Artificial Magnetic Conductor (AMC) is the name that was established for the complex EBG structures (the "mushroom" periodic surface above a conducting ground) initially presented as High Impedance Structures (HIS) in [11] because of the specific property that the reflection of plane waves from such a surface occurs such that the reflected wave is in phase with the incident wave. This property would be exhibited by a magnetic conductor (dual of the perfect electric conductor).

Different types of AMC reflectors have been investigated since the publication of [11], eg. in [13] a comparative study was performed on four types of AMC surfaces, including the "mushroom" EBG, uniplanar compact EBG, Peano curve, and Hilbert curve. The study shows that the "mushroom" surface has the best bandwidth of the four types considered. Another popular type of AMC is the Jerusalem Cross-based structure [14], but our own simulations have shown that the bandwidth of this structure (implemented on the same substrate) is also substantially less than that of the "mushroom" AMC. A modification of the "mushroom" AMC was proposed in [15]the vias were removed between the patches and the ground because vias complicate the fabrication of AMC surfaces - the effect of this was negligible on the AMC performance of the structure for normal incidence, but the EBG typically shifts to higher frequencies. In [15] the AMC and EBG characteristics of this new patch array AMC were investigated and a new technique presented to tailor the spectral position of the AMC operation and the EBG.

Because of the bandwidth advantage, and the simplicity of fabrication it was decided to use the patch array AMC proposed in [15] for the antenna in this paper - a unit cell of the proposed metasurface is shown in Fig. 2. The performance of an infinite repetition of this basic cell has been simulated using CST Microwave Studio applying the proper boundary conditions (PEC's on the planes normal the polarization of the incident field, and PMC's on the planes parallel to the polarization of the incident field) to the unit cell and under normal incidence. The total structure thus comprises of a metal backed dielectric substrate with an array of metal square patches arranged in a periodical form. Design by repetitive analysis resulted in final dimensions for the structure: $A=25$ $\mathrm{mm}, B=24 \mathrm{~mm}$ (which implies $g=A-B=1 \mathrm{~mm}$, referring to Fig. 1) and substrate thickness $T=3.048 \mathrm{~mm}\left(\mathrm{RO} 4003, \varepsilon_{\mathrm{r}}=\right.$ $3.38, \tan \delta=0.0021)$. The design aim was to get zero reflecting phase at a resonant frequency of $2.45 \mathrm{GHz}$.
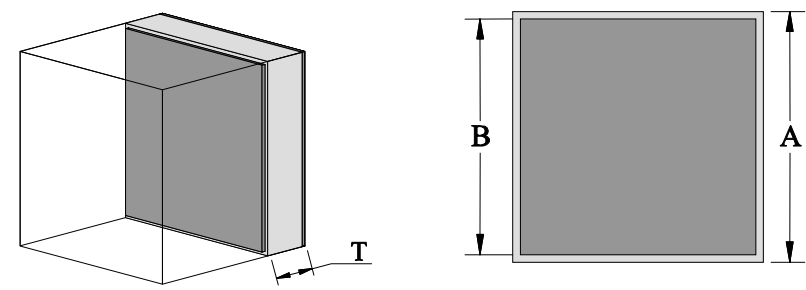

Fig. 2. 3D and front view of unit cell for determining of the resonant frequency (with $0^{\circ}$ reflecting phase) of the AMC surface.

The reflection phase of the unit cell is shown in Fig. 3. If one includes a metallic via embedded inside the dielectric substrate connecting the metal square patch to the ground plane the well known "mushroom" type AMC is obtained [10, 11]. This structure was also analyzed and the result is also shown in Fig. 3. The reflection phase response of the patch $\mathrm{AMC}$ and the "mushroom" AMC were found to be very similar. The reflection phase of the structure is $0^{\circ}$ at $2.45 \mathrm{GHz}$ and between $\pm 90^{\circ}$ in the frequency range from $2.28 \mathrm{GHz}$ to $2.64 \mathrm{GHz}$ (approximately over a 15\% bandwidth). The magnitude of the reflection coefficient is close to +1 . The bandwidth of the structure can be improved if one uses a thicker substrate or a substrate with a lower permittivity, but the overall antenna structure will then be thicker (less lowprofile), or the unit element size will have to be increased.

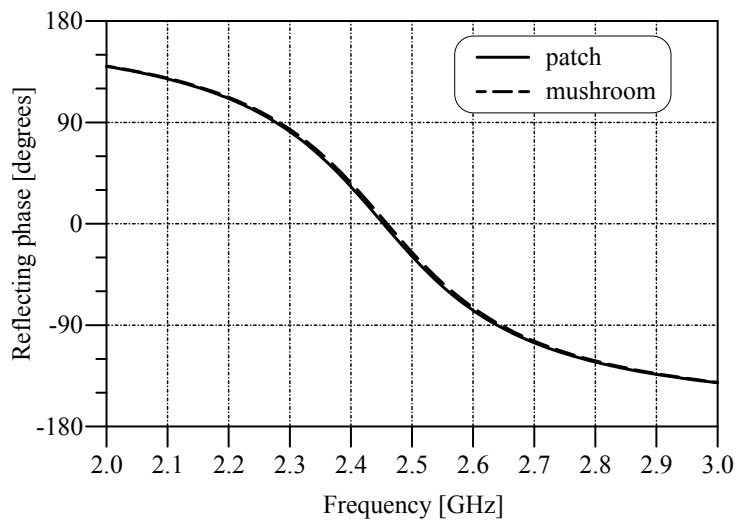

Fig. 3. The simulated reflecting phase response for a patch $\mathrm{AMC}$ and a "mushroom" AMC. In both cases the dimensions were $A=25 \mathrm{~mm}, B=24 \mathrm{~mm}$ $(g=1 \mathrm{~mm})$ and $T=3.048 \mathrm{~mm}$ (on substrate RO4003, $\varepsilon_{\mathrm{r}}=3.38, \tan \delta=$ $0.0021)$. 
Final Author version. Paper published in IEEE Transactions on Antennas \& Propagation. Feb. 2012, Vol. 60. Issue 2, pp. 735-742

\section{Cavity-Backed Slot Antenna Design}

The CPW-fed cavity-backed slot antenna loaded with the AMC reflector designed in the previous section (see Fig. 1) was designed to operate in the WLAN frequency band (2.4 $2.484 \mathrm{GHz}$ ). The radiating element basically consists of two half-wavelength slots radiating in phase and in close proximity to each other - each slot fed by one of the slotlines of the CPW feed line. It is sometimes referred to as a fullwavelength printed slot radiator, and this type of element (without the AMC reflector) has previously been characterized in $[16,17]$. The design of the final antenna was performed with the assistance of CST Microwave Studio. The radiating slot element substrate was chosen as Rogers RO4003, with $\varepsilon_{\mathrm{r}}=3.38, \tan \delta=0.0021$ and $t=1.524 \mathrm{~mm}$. The antenna design process involved the determination of suitable values for some inter-dependant parameters, specifically gap, $L_{g}$ and $W_{g}$, and $L$ and $W$ (all of them indicated in Fig. 1).

\section{A. Gap - The Distance Between the Slot Substrate and the AMC Reflector}

Simulations indicated that the bandwidth of the antenna begins to reduce dramatically for gap $<3 \mathrm{~mm}$. This probably happens because the AMC reflector performance begins to change drastically as the slot substrate comes closer in proximity to the AMC structure (the AMC reflector was designed for an air medium in front of the reflector). A final value of gap $=3 \mathrm{~mm} \approx 0.025 \lambda_{0}$ was used. The overall height of the final antenna was $7.662 \mathrm{~mm} \approx 0.063 \lambda_{0}$, with $\lambda_{0}$ the freespace wavelength at $2.45 \mathrm{GHz}$.

\section{B. $L_{g}$ and $W_{g}$ - The Lateral Cavity Dimensions}

$L_{g}$ and $W_{g}$, (the lateral cavity dimensions of the antenna), and also the number of patches used to construct the AMC reflector were chosen to minimize the power radiated from the sides of the cavity structure, which contribute towards high sidelobes and a high backlobe in the radiation pattern. This effective EBG performance was found to be strongly influenced by the lateral cavity dimensions (for a specific value of gap). The EBG stop band was initially determined using the method proposed in [10], where the slot is removed from the top conducting surface (and replaced with a PEC), and two ports on either side of the parallel plate cavity are defined, and the transmission coefficient then determined. The two remaining sides of the cavity were designated as perfect magnetic walls. The results showed that the stop band did not coincide with the AMC reflector band, with high transmission levels predicted within the $2.4-2.5 \mathrm{GHz}$ band. Upon further investigation it was soon realized that this method does not necessarily determine the EBG performance of our finite cavity structure very accurately - the excitation of parallel plate modes by a slot in the top conducting surface of the finite cavity structure might be very different to that of the structure with the magnetic walls as described in [10]. A new EBG performance test set-up was devised, consisting of a dielectric-filled waveguide feeding the finite parallel plate cavity formed by the slot conducting surface, the antenna substrate and the AMC reflector, as shown in Fig. 4. The assumption is that the waveguide aperture will excite parallel plate modes in the cavity very similar to a slot. The simulated reflection coefficient of the waveguide port will then be indicative of the EBG performance of the structure.

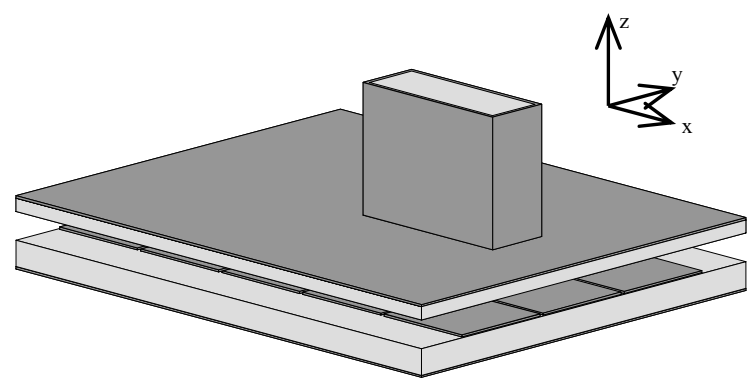

Fig. 4. EBG performance test set-up consisting of a dielectric-filled rectangular waveguide (in the position of one of the radiating slots) feeding the parallel plate cavity formed by the finite slot ground plane, antenna substrate and $\mathrm{AMC}$ reflector.

The dimensions and location of the waveguide feed were chosen to be the same as one half-wavelength of the radiating slot element, and then suitable compact cavity dimensions were determined through repetitive analysis. The EBG performance of three representative cases (see Fig. 5) are discussed: (a) Case\#1 $-5 \times 5$ patch array $\operatorname{AMC}\left(L_{g}=W_{g}=\right.$ $125 \mathrm{~mm})$, (b) Case \#2 $-5 \times 4$ patch array $\operatorname{AMC}\left(L_{g}=125 \mathrm{~mm}\right.$, $\left.W_{g}=100 \mathrm{~mm}\right)$, and (c) Case\#3 $-5 \times 3$ patch array AMC $\left(L_{g}=\right.$ $125 \mathrm{~mm}, W_{g}=100 \mathrm{~mm}$ ). The basic difference between Case\#1 and Case\#2 is the cavity dimension in the y-direction. Case\#2 and Case\#3 have exactly the same cavity dimensions, but the patch array sizes are different in the two cases, and in all cases gap $=3 \mathrm{~mm}$ was used.

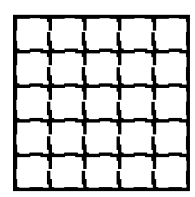

(a)

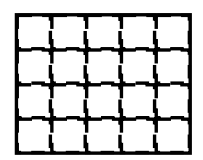

(b)

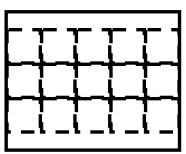

(c)
Fig. 5. Three different test cases for which the EBG performance were investigated; (a) Case\#1 $-5 \times 5$ patch array AMC $\left(L_{g}=W_{g}=125 \mathrm{~mm}\right)$, (b) Case\#2 $-5 \times 4$ patch array $\operatorname{AMC}\left(L_{g}=125 \mathrm{~mm}, W_{g}=100 \mathrm{~mm}\right)$, and (c) Case\#3 $-5 \times 3$ patch array AMC $\left(L_{g}=125 \mathrm{~mm}, W_{g}=100 \mathrm{~mm}\right)$.

The waveguide port was excited with a dominant waveguide mode and the reflection coefficient calculated. From Fig. 6 one can see that the difference in the reflection coefficient is significant for Case\#1 and Case\#2 - illustrating the significance of the cavity dimensions. The difference in reflection coefficient is less significant between Case\#2 and Case\#3, for different array sizes but the same cavity dimensions. Case\#3 has the largest reflection coefficient over the $2.4-2.5 \mathrm{GHz}$ band, which is an indication that the waveguide aperture radiates into a very high impedance for 
Final Author version. Paper published in IEEE Transactions on Antennas \& Propagation. Feb. 2012, Vol. 60. Issue 2, pp. 735-742

that particular structure, thus limiting significant leakage of power between the cavity side walls. Case\#3 was chosen for the final antenna design $-L_{g}=125 \mathrm{~mm} \approx 1.02 \lambda_{0}, W_{g}=100$ $\mathrm{mm} \approx 0.82 \lambda_{0}$, and a $5 \times 3$ patch array AMC. For a compact cavity-backed slot antenna like this the cavity dimensions are the primary parameters that have to be determined to ensure minimum energy leakage. Because of the small cavity size and the close proximity of the slots to the cavity edges, and hence the small surface area around the slot where an EBG surface can be employed, the mode suppression by the periodic structure of the AMC reflector does not seem to play a significant role. For larger structures like those in $[7,9,10]$, the mode suppression of the periodic structure of the AMC will play a more significant role, and the newly devised EBG test set-up will also be suitable for future designs of such structures.

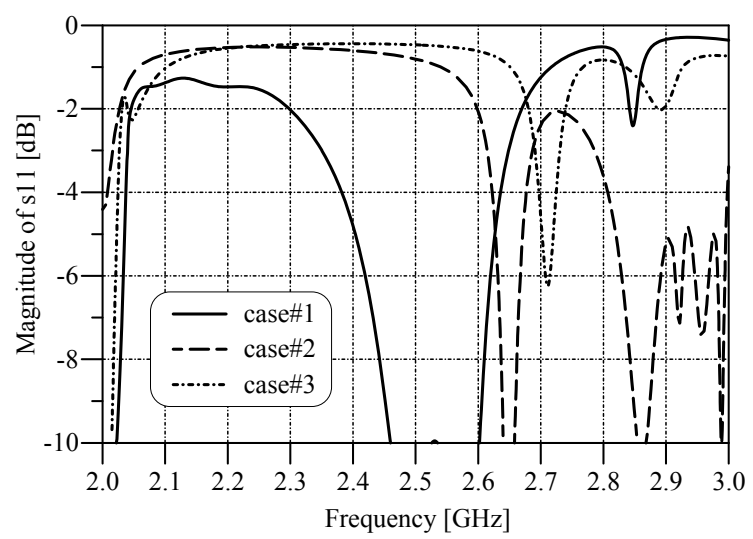

Fig. 6. Simulated magnitude of $s_{11}$ for the waveguide port - for the three test cases shown in Fig. 5.

\section{L and $W-$ The Slot Dimensions}

$L$ and $W$, the slot length and width were determined that will provide an impedance match to a $50 \Omega \mathrm{CPW}$ feed line. For the $50 \Omega \mathrm{CPW}$ feed line the gap between the slotlines was determined as $c_{c p w}=4.2 \mathrm{~mm}$ and the slotline width as $s_{c p w}=$ $0.4 \mathrm{~mm}$. The slot width primarily determines the radiation resistance, and the slot length primarily determines the resonant frequency. The final antenna design slot dimensions were determined as $L=78.2 \mathrm{~mm}$ and $W=14.0 \mathrm{~mm}$.

\section{REFERENCE ANTENNA DESIGN}

For the purpose of gain and bandwidth comparisons a reference antenna without an AMC reflector was also designed using the same substrate and the same lateral dimensions $L_{g}=125 \mathrm{~mm}$ and $W_{g}=100 \mathrm{~mm}$. If the final antenna design slot dimensions as determined in the previous section are used and the antenna simulated without the AMC reflector, the radiation resistance of the slot is approximately doubled, and the resonant frequency increases. The final reference slot dimensions can be obtained by decreasing the slot width (to $W=6.5 \mathrm{~mm}$ ), for a radiation resistance $50 \Omega$, and by increasing the slot length (to $L=88 \mathrm{~mm}$ ), for resonance at $2.45 \mathrm{GHz}$. The reference antenna above an infinite PEC reflector spaced at gap $=3 \mathrm{~mm}$ was also analyzed. The antenna was still reasonably well matched, but the E-plane radiation patterns were seriously distorted because of significant energy leakage from the sides of the antenna. To confirm the EBG test procedure (in Fig. 4) was performed for the antenna above the PEC reflector, and the waveguide port reflection coefficient is shown in Fig. 7 - the level of $-3 \mathrm{~dB}$ at the design frequency is a clear indication of significant energy leakage.

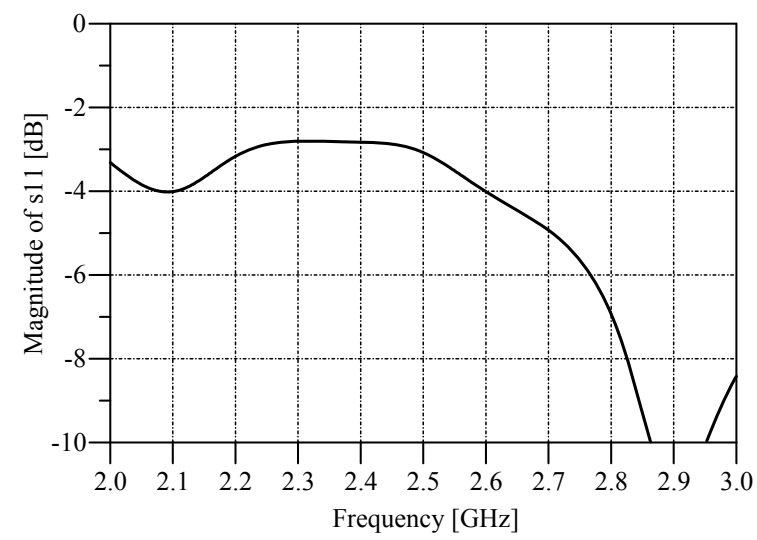

Fig. 7. Simulated magnitude of $s_{11}$ for the waveguide port - for an infinite PEC reflector spaced at $g a p=3 \mathrm{~mm}$.

\section{Results of Final Design AND Discussion}

To validate the simulations the slot antenna with the AMC reflector was manufactured and measured. A photograph of the final antenna is shown in Fig. 8. The different layers were fixed with four plastic screws with $3 \mathrm{~mm}$ spacers.

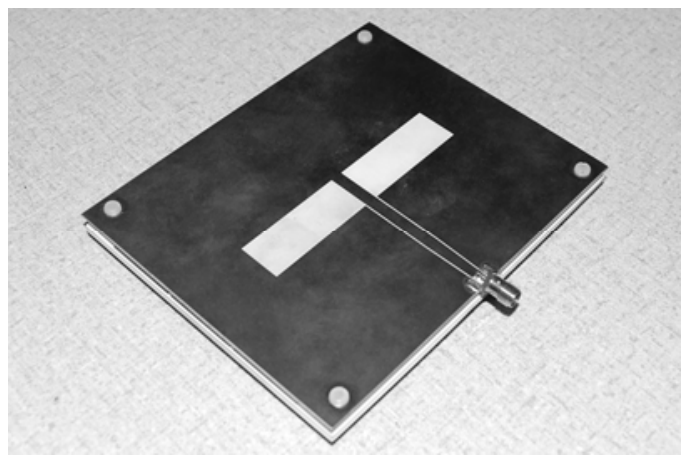

Fig. 8. Photograph of the manufactured final antenna design.

A comparison between the simulated and measured reflection coefficient is shown Fig. 9. The two sets of data correspond well, and the impedance bandwidth $(-10 \mathrm{~dB}$ return loss) was found to be close to $5 \%$. It has to be mentioned that the impedance bandwidth of the reference slot antenna without any reflector was more than $20 \%$. The much lower bandwidth achieved with the reflector is due to the limited 
Final Author version. Paper published in IEEE Transactions on Antennas \& Propagation. Feb. 2012, Vol. 60. Issue 2, pp. 735-742

bandwidth of the AMC surface in terms of reflection phase. The overall bandwidth of the final antenna roughly corresponds to the bandwidth of the AMC surface where the reflection phase is between $\pm 45^{\circ}$. The electromagnetic band gap of the structure is significantly broader (define as $2.2-$ 2.6 GHz, from Fig. 6), so if one can improve the reflection phase bandwidth an antenna with better overall bandwidth will be possible.

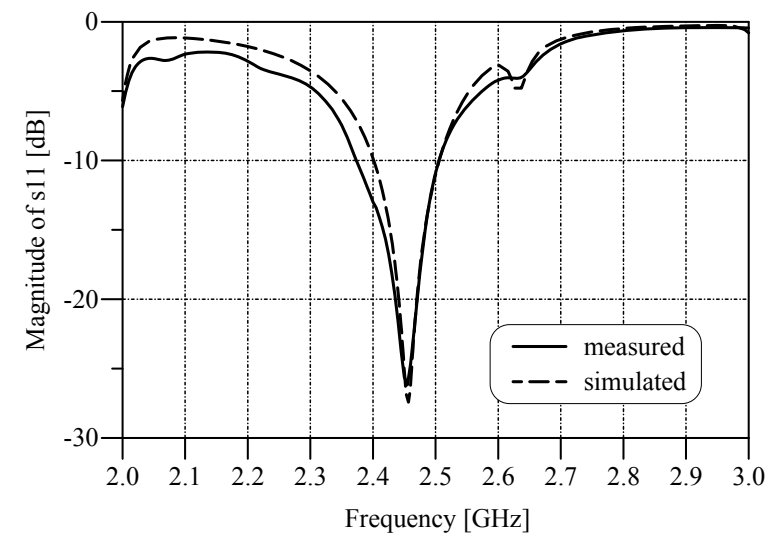

Fig. 9. Simulated and measured reflection coefficient $\left(s_{11}\right)$ for the final antenna design.

The E- and H-plane radiation patterns of the final antenna design were measured at $2.45 \mathrm{GHz}$. The simulated and measured patterns are shown in Figures 10 and 11. The crosspolarization was found to be very low (more than $20 \mathrm{~dB}$ down), and the front-to-back ratio acceptable - in the region of $18 \mathrm{~dB}$. To improve the front-to-back ratio the final antenna design was mounted on a back plate with a size $1.5 \lambda_{0} \times 1.5 \lambda_{0}$ $=185 \mathrm{~mm} \times 185 \mathrm{~mm}$. Fig. 12 shows a photograph of the final antenna design mounted on the back plate. Simulations have shown that the back plate added to the structure does not affect the EBG properties or the matching of the antenna significantly - the $\mathrm{s}_{11}$ was still lower than $-10 \mathrm{~dB}$ for the entire WLAN band. The back plate does however change the radiation direction of the small amount of leaked energy from the cavity sidewalls - with less energy being radiated towards the back and sides of the antenna. The simulated and measured E- and H-plane radiation patterns are shown in Figures 13 and 14. The front-to-back ratio improved to about $25 \mathrm{~dB}$.

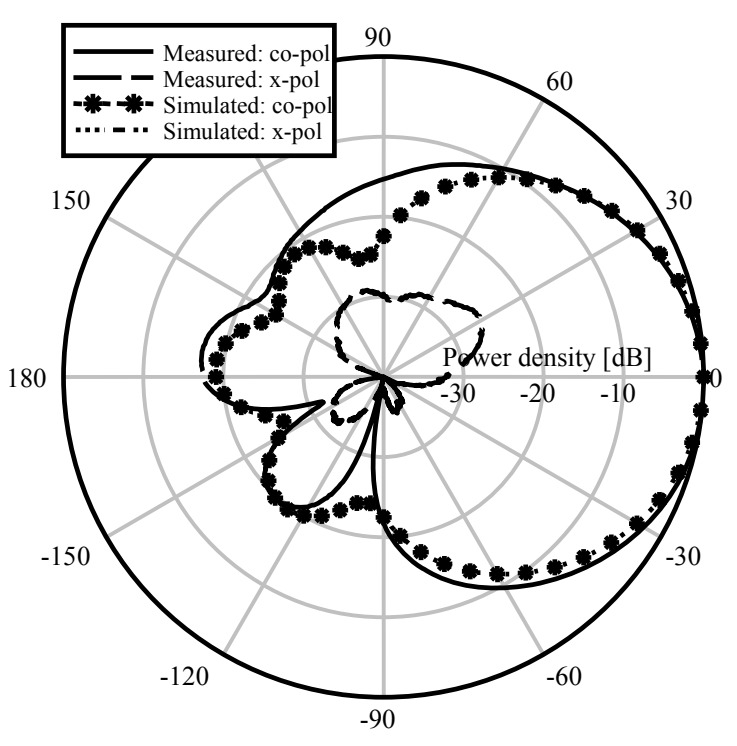

Fig. 10. Simulated and measured E-plane radiation patterns for the final antenna design.

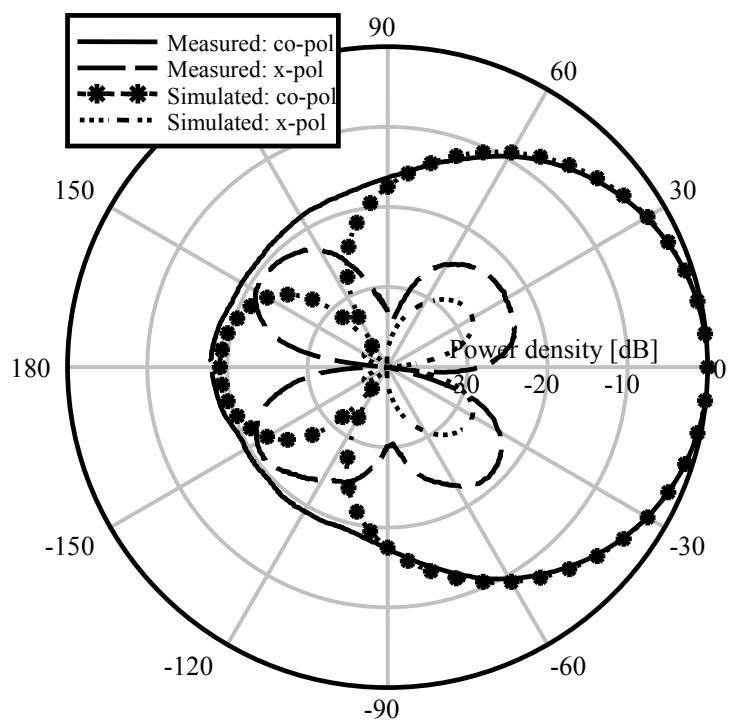

Fig. 11. Simulated and measured H-plane radiation patterns for the final antenna design.

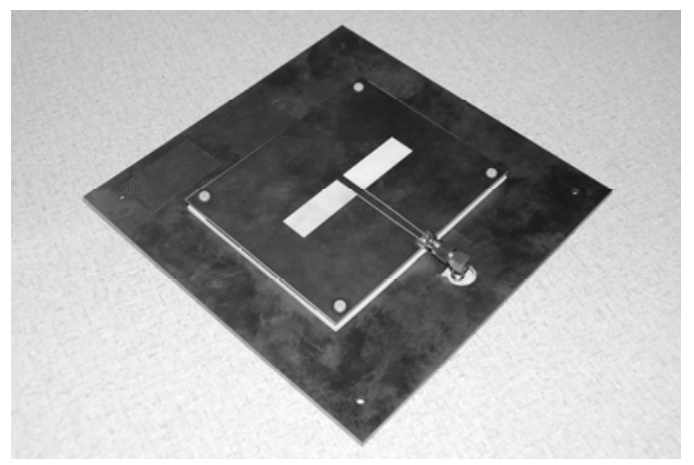

Fig. 12. Photograph of the manufactured final antenna design mounted on a $1.5 \lambda_{0} \times 1.5 \lambda_{0}$ back plate. 
Final Author version. Paper published in IEEE Transactions on Antennas \& Propagation. Feb. 2012, Vol. 60. Issue 2, pp. 735-742

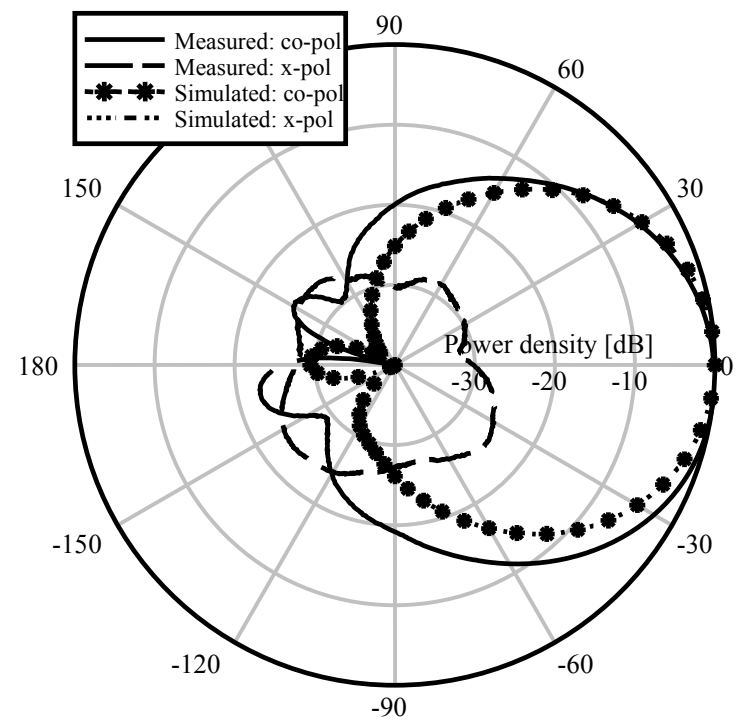

Fig. 13. Simulated and measured E-plane radiation patterns for the final antenna design mounted on a $1.5 \lambda_{0} \times 1.5 \lambda_{0}$ back plate.

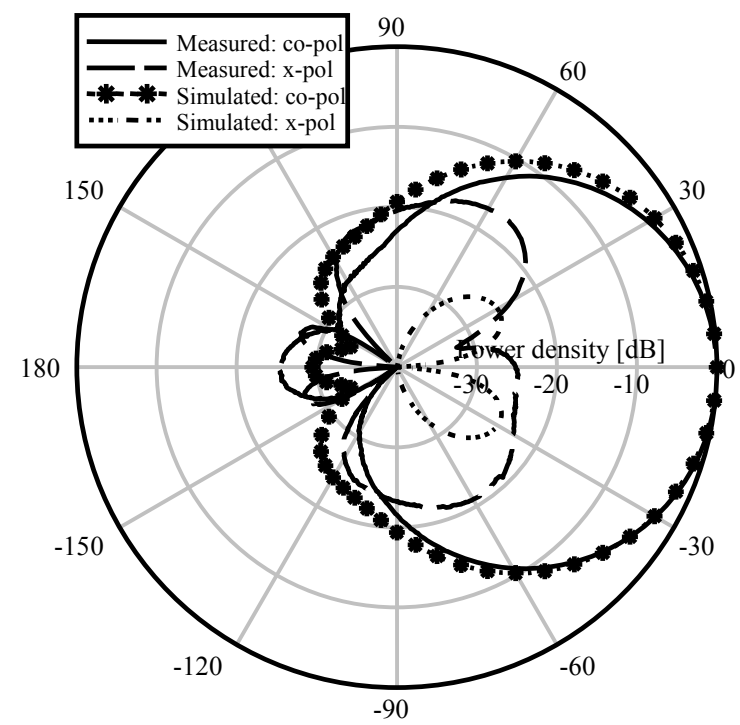

Fig. 14. Simulated and measured H-plane radiation patterns for the final antenna design mounted on a $1.5 \lambda_{0} \times 1.5 \lambda_{0}$ back plate.

The realized gain was simulated and measured for the slot antenna without an AMC reflector, the final antenna design (with an AMC reflector and lateral dimensions of $125 \mathrm{~mm} \times$ $100 \mathrm{~mm}$ ) and the same design mounted on the $1.5 \lambda_{0} \times 1.5 \lambda_{0}$ back plate, and the results are shown in Figure 15. Good correlation between the simulated and measured values can be observed. The gain of the final antenna design is approximately $3 \mathrm{~dB}$ higher than the gain of the antenna without a reflector, and the gain improved by another $\mathrm{dB}$ for the final design mounted on the larger back plate. The measured gain of the final antenna mounted on the back plate varied between 9.8 and $10.3 \mathrm{~dB}$ within the $2.4-2.5 \mathrm{GHz}$ band. Also evident in Fig. 15 is the more constant gain over a wider bandwidth (close to $6 \mathrm{dBi}$ ) of the reference antenna which has a much larger bandwidth because of the absence of the effect of the AMC reflector.

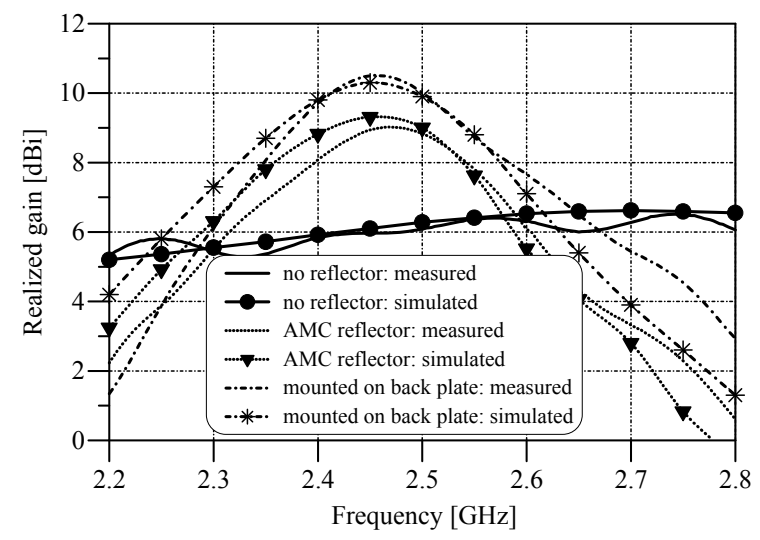

Fig. 15. Simulated and measured antenna gain for the reference slot antenna without a reflector, the final antenna design with an AMC reflector, and the final antenna design with an AMC reflector mounted on a $1.5 \lambda_{0} \times 1.5 \lambda_{0}$ back plate.

The simulated radiation and total efficiencies of the final antenna mounted on the $1.5 \lambda_{0} \times 1.5 \lambda_{0}$ back plate are shown in Fig. 16 - the radiation efficiency is better than $95 \%$ and the total efficiency better than $85 \%$ for the entire $2.4-2.5 \mathrm{GHz}$ band.

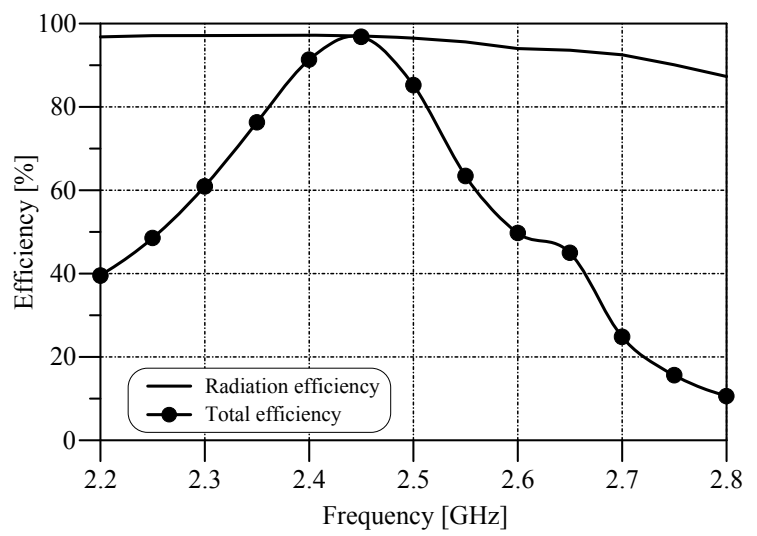

Fig. 16. Simulated radiation and total efficiencies of the final antenna mounted on the $1.5 \lambda_{0} \times 1.5 \lambda_{0}$ back plate.

\section{CONCLUSIONS}

A low profile CPW-fed cavity-backed printed slot antenna is presented with uni-directional radiation properties. The slot antenna radiates above a closely spaced artificial magnetic conducting reflector consisting of an array of rectangular patches, a substrate and an electric ground plane. The basic AMC reflector design was based on an infinite structure, but the final dimensions of the metamaterial based reflector cavity was determined on the basis of a newly devised EBG performance test for the finite structure. The EBG performance was evaluated with an equivalent waveguide feed in place of one half-wavelength section of the slot antenna. From the waveguide reflection coefficient one can 
Final Author version. Paper published in IEEE Transactions on Antennas \& Propagation. Feb. 2012, Vol. 60. Issue 2, pp. 735-742

determine the frequency band where minimum energy will be lost due to unwanted modes propagating between the parallel plates and radiating from the cavity sides. Experimental results for the final antenna design (with a size of $1.02 \lambda_{0} \times$ $\left.0.82 \lambda_{0} \times 0.063 \lambda_{0}\right)$, mounted on $1.5 \lambda_{0} \times 1.5 \lambda_{0}$ back plate, exhibit a $5 \%$ impedance bandwidth, maximum gain in excess of $10 \mathrm{dBi}$, low cross-polarization, and a front-to-back ratio of approximately $25 \mathrm{~dB}$. This antenna is simple to manufacture using simple photolithography (no vias or solid sidewalls are necessary), and can be used as a flush-mounted single radiator. This low-profile antenna with relatively high gain could be a good candidate for a $2.4 \mathrm{GHz}$ WLAN application. Future investigations will include the design of arrays of printed slots above an AMC reflector, for which the newly devised EBG test procedure will be a valuable design tool.

\section{REFERENCES}

[1] A. Vallecchi and G. Biffi Gentili, "Microstrip-fed slot antennas backed by a very thin cavity," Microwave and Optical Tech. Letters, vol. 49, no. 1, pp. 247-250, Jan. 2007

[2] C. Löcker, T. Vaupel and T.F. Eibert, "Radiation efficient unidirectional low-profile slot antenna elements for X-band applications," IEEE Trans. Antennas Propagat., vol. 53, no. 8, pp. 2765-2768, Aug. 2005.

[3] M. Qiu, M. Simcoe, and G.V. Eleftheriades, "Radiation efficiency of printed slot antennas backed by a ground reflector," 2000 IEEE AP-S Symp. Dig., pp. 1612-1615.

[4] J. P. Jacobs, "Self and mutual admittance of CPW-fed slots on conductor-backed two-layer substrate," Microwave and Optical Tech. Letters, vol. 49, no. 11, pp. 2798-2802, Nov. 2007.

[5] J.P. Jacobs, J. Joubert, and J.W. Odendaal, "Radiation efficiency and impedance bandwidth of conductor-backed CPW-fed broadside twin slot antennas on two-layer dielectric substrate," IEE Proc-Microwave Antennas Propag., vol. 150, pp. 185-190, 2003.

[6] M. Qiu and G.V. Eleftheriades, "Highly efficient unidirectional twin arcslot antennas on electrically thin substrates," IEEE Trans. Antennas Propagat., vol. 52, no. 1, pp. 53-58, Jan. 2004.

[7] J. Y. Park, C.-C. Chang, Y. Qian, and T. Itoh, "An improved low-profile cavity-backed slot antenna loaded with 2D UC-PBG reflector," Proc. IEEE Int. Symp. Antennas and Propagation, Boston, MA, pp. 194-197, Jul. 2001.

[8] A.O. Karilainen, J. Vehmas, O. Luukkonen, and S.A. Tretyakov, "Highimpedance-surface-based antenna with two orthogonal radiating modes," IEEE Antennas and Wireless Propagat. Lett., vol. 10, pp. 247-250, 2011

[9] J. D. Shumpert, W. J. Chappell, and L. B. Katehi, "Parallel-plate mode reduction in conductor backed slots using electromagnetic bandgap substrates," IEEE Trans. Microw. Theory Tech., vol. 47, no. 11, pp. 2099-2104, Nov. 1999.

[10] F. Elek, R. Abhari and G.V. Eleftheriades, "A unidirectional ring-slot antenna achieved by using an electromagnetic band-gap surface," IEEE Trans. Antennas Propagat., vol. 53, no. 1, pp. 181-190, Jan. 2005.

[11] D. Sievenpiper, L. Zhang, R. F. J. Broas, N. G. Alexopoulos, and E. Yablonovitch, "High-impedance electromagnetic surfaces with a forbidden frequency band," IEEE Trans. Microw. Theory Tech., vol. 47, no. 11, pp. 2059-2074, Nov. 1999.

[12] G. Niyomjan and Y. Huang, "A suspended microstrip fed slot antenna on high impedance surface structure," Proc of EuCAP, Nice, France, Nov. 2006.

[13] J. R. Sohn, K. Y. Kim, and H.-S. Tae, "Comparative study on various artificial magnetic conductors for low-profile antenna," PIER, vol. 61, pp. 27-37, 2006 .

[14] M. Hosseini and M. Hakkak, "Characteristics estimation for Jerusalem Cross-based artificial magnetic conductors," IEEE Antennas and Wireless Prop. Letters, vol. 7, pp. 58-61, 2008.

[15] G. Goussetis, A. Feresidis, and J.C. Vardaxoglou, "Tailoring the AMC and EBG Characteristics of Periodic Metallic Arrays Printed on
Grounded Dielectric Substrate," IEEE Trans. Antennas Propagat., vol. 54, no. 1, pp. 82-89, Jan. 2006.

[16] A. Nešić, "Slotted antenna array excited by a coplanar waveguide," Electronics Letters, vol.18, pp. 275-276, 1982.

[17] H.-C. Liu, T.-S. Horng, and N.G. Alexopoulos, "Radiation of printed antennas with a coplanar waveguide feed," IEEE Trans. Antennas Propagat., vol. 43, no. 10, pp. 1143-1148, Oct. 1995.

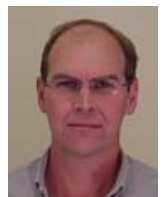

Johan Joubert (M'86-SM'05) received the B.Eng, M.Eng and $\mathrm{PhD}$ degrees in Electronic Engineering from the University of Pretoria, Pretoria, South Africa, in 1983, 1987 and 1991 respectively.

From 1984 to 1988 he was employed as a Research Engineer at the Council for Scientific and Industrial Research, Pretoria. In 1988 he joined the Department of Electrical and Electronic Engineering at the University of Pretoria, where he is currently a Professor of Electromagnetism. From July to December 1995 he was Visiting Scholar with the Department of Electrical and Computer Engineering, California State University, Northridge, USA. From July to December 2001 he was Visiting Scientist at Industrial Research Laboratories in Wellington, New Zealand. From July to December 2006 he was Visiting Scholar at the Institut für Höchstfrequenztechnik und Elektronik, Universität Karlsruhe (TH), Germany, and from July to September 2010 he visited Loughborough University in the UK for a collaborative research project on metamaterials. His research interests include antenna array design and computational electromagnetism. Prof. Joubert is a registered Professional Engineer in South Africa.

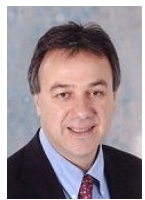

J.(Yiannis) C. Vardaxoglou (M'87) completed his B.Sc. in mathematical physics (1982) and his Ph.D. in Electronics (1985) at the University of Kent, UK.

He joined Loughborough University as a lecturer in 1988 and was promoted to Senior Lecturer in 1992 and Professor or Wireless Communications in 1998. He is currently Dean of the School of Electronic, Electrical and Systems Engineering at Loughborough University. He established the Wireless Communications Research (WiCR) group at Loughborough University and heads the Centre for Mobile Communications Research. He has pioneered research, design and development of frequency selective surfaces (FSS) for communication systems, Metamaterials and low SAR antennas for mobile telephony and has commercially exploited a number of his innovations. He has served as a consultant to various industries, holds 6 patents and is the Technical Director of Antrum Ltd (a University spinout company). He has attracted research funding from industry and has been awarded 18 EPSRC research grants. He has published over 160 refereed journals and conference proceeding papers and has written a book on FSS.

Prof. Vardaxoglou was Chairman of the Executive Committee of the IET's Antennas and Propagation Professional Network in the UK and chaired the IEEE's distinguish lecturer program of the Antennas and Propagation Society (APS) for 5 years. He was the General Chair of EuCAP'2007. He has chaired numerous IEE//IET events and has been on the Steering Committee of the European Conference on Antennas and Propagation, EuCAP since 2006. He founded the Loughborough Antennas and Propagation Conference (LAPC), which has been running since 2005 .

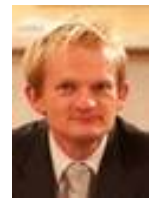

William G. Whittow received the B.Sc. in physics from The University of Sheffield, Sheffield, U.K in 2000 and his Ph.D in the EEE Department also at Sheffield in 2004.

$\mathrm{He}$ is currently employed as a Research Associate at Loughborough University, UK. He has published over 60 peer reviewed journal and conference papers in topics related to electromagnetic materials, wearable antennas, VHF antennas, Specific Absorption Rate, FDTD, bioelectromagnetics, phantoms and Genetic Algorithms.

Since 2007, Dr. Whittow has been the Coordinating Chair of the Loughborough Antennas \& Propagation Conference (LAPC).

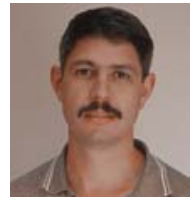

Johann W. Odendaal (M'90-SM'00) received the B.Eng, M.Eng, and Ph.D degrees in electronic engineering from the University of Pretoria, Pretoria, South Africa, in 1988, 1990, and 1993, respectively.

From September 1993 to April 1994, he was a Visiting Scientist with the ElectroScience Laboratory at the Ohio 
Final Author version. Paper published in IEEE Transactions on Antennas \& Propagation. Feb. 2012, Vol. 60. Issue 2, pp. 735-742

State University. From August to December 2002, he was a Visiting Scientist with CSIRO Telecommunications and Industrial Physics in Australia. Since May 1994, he has been with the University of Pretoria, where he is currently a Full Professor. His research interests include electromagnetic scattering and radiation, compact range measurements, and signal processing. He is also Director of the Centre for Electromagnetism at the University of Pretoria.

Prof. Odendaal is a member of the Antenna Measurement Techniques Association (AMTA) and is registered as a Professional Engineer in South Africa. 